\title{
STABILITY AND FINITE SUM OF WILSON FRAMES
}

\author{
SHIV KUMAR KAUSHIK AND SUMAN PANWAR ${ }^{\dagger}$
}

\author{
Date of Receiving : : 13.04. 2016 \\ Date of Revision : 25.06. 2016 \\ Date of Acceptance : $\quad$ 27. 06. 2016
}

\begin{abstract}
Wilson frames have been defined in [10]. In this paper we discuss about finite sum of Wilson frames in case of similar window functions. A necessary and sufficient condition is obtained under which finite sum of Wilson frames is a Wilson frame. Finally, a sufficient condition under which finite sum of Wilson Bessel sequences is a Bessel sequence for $L^{2}(\mathbb{R})$ in terms of their frame bounds is obtained.
\end{abstract}

\section{Introduction}

Frames for Hilbert spaces were introduced by Duffin and Schaeffer[5] in 1952. Daubechies, Meyer and Grossmann [3],in 1986, reintroduced frames and observed that frames can be used to find series expansions of functions in $L^{2}(\mathbb{R})$. Time-frequency representations play a fundamental role in many practical applications as they provide localized information of signals in time and frequency domains. Series representations in terms of frame capture such information at prescribed discrete points in timefrequency plane. Gabor frames find wide applications in time-frequency analysis, signals processing, etc $[2,7,8,9]$.

However, in view of the Balian-Low Theorem, Gabor frames which are Reisz basis for $L^{2}(\mathbb{R})$ do not possess good localization properties in time and frequency simultaneously. This drawback was overcome by a Wilson system defined by Daubechies, Jaffard and Journe in [4]. K. Bittner in [1] generalised the system defined by Daubechies, Jaffard and Journe which also overcame the drawbacks of the BalianLow Theorem. In this paper we discuss stability of Wilson frames and finite sum of Wilson frames in case of same window functions.

2010 Mathematics Subject Classification. 42C15, 94A12.

Key words and phrases. Wilson frames, Gabor frames, Wilson Bessel sequence, Gabor Bessel sequence.

Communicated by. A. Askari Hemmat

${ }^{\dagger}$ Corresponding author. 